\title{
Femtocell interference analysis based on the development of system-level LTE simulator
}

\author{
Sueng Jae Bae1, Young Min Kwon ${ }^{1}$, Mi-Young Lee², Bon Tae Koo² and Min Young Chung ${ }^{\text {** }}$
}

\begin{abstract}
Although femtocell can confer various benefits to both operators and users, many technical issues, such as interference coordination, network synchronization, self-configuration, and self-optimization must be considered to effectively deploy femtocells in real environments. In this article, we develop a system-level simulator in order to evaluate performance of long-term evolution (LTE)-femtocell systems. The simulator consists of five functional modules which realize packet scheduling and call admission control functionalities and model various wireless channel effects, real and non-real time traffics, and user mobility. These modules are managed by operational modules that follow event-driven programming. In addition, the simulator adopts a graphical user interface (GUI) that facilitates configuration, management, and observation of simulations in real time. Based on the simulator, we analyze effects of signal interference between macro- and femtocells under four scenarios. From the results, we confirm that the developed LTE-femtocell simulator can effectively support performance evaluation and analysis on various femtocell deployment scenarios.
\end{abstract}

Keywords: Deployment scenarios, Event-driven programming, Femtocell, Interference analysis, LTE, Simulator development

\section{Introduction}

Recently, demands of customers for wireless data communications in cellular networks are rapidly increasing as fascinating mobile devices, e.g., smart phone, tablet device, and ultra-mobile personal computer (UMPC), and mobile applications, e.g., mobile Internet protocol television (IPTV), high-definition video conference, and voice over IP (VoIP), have been introduced. For example, traffics for wireless data communications have increased by $5000 \%$ over the past three years in AT\&T networks [1]. Moreover, customers expect to be provided with enhanced quality of services since they spend more communication expenses to telecommunications companies for wireless data communications than they do for voiceonly services.

In indoor environments, channel quality between the cellular base station and mobile node may be low due to many walls and obstacles. Thus, wireless communication for indoor environments requires more wireless

*Correspondence: mychung@ece.skku.ac.kr

${ }^{1}$ College of Information and Communication Engineering, Sungkyunkwan University, Suwon, 440-746, Republic of Korea

Full list of author information is available at the end of the article resources including time, bandwidth, and transmit power, to guarantee required service quality of customers. Nevertheless, shortage of wireless resource in cellular networks will be accelerated, since more than $60 \%$ of voice traffic and $90 \%$ of data traffic are expected to be generated indoors [2].

It is undeniable that extra network infrastructure is required to expand the capacity of cellular networks. The use of femtocells, which are overlaid over the traditional macrocell based cellular networks is a key topic related to the deployment of new infrastructure in a cost-effective manner [3-5]. A femtocell is smallsized cellular base station with low-power and low-cost characteristics. It can be deployed wherever wired IP access is possible by either the service providers or the customers. Since this inexpensive femtocell is usually deployed indoors and connected to the backhaul through generic IP access, such as digital subscriber line (DSL), cable modem, and fiber-to-the-home (FTTH), it can help cellular networks to cost-efficiently secure wireless resources and customers to use wireless data communications with low power and high data rates using

\section{望 Springer}

(c) 2012 Bae et al.; licensee Springer. This is an Open Access article distributed under the terms of the Creative Commons Attribution License (http://creativecommons.org/licenses/by/2.0), which permits unrestricted use, distribution, and reproduction in any medium, provided the original work is properly cited. 
existing cellular devices. Despite these advantages, several technical issues should be considered for efficient femtocell deployment, e.g., signal interference coordination, resource allocation and management, access strategy, network synchronization, self-organization and optimization, and handover between macro- and femtocells. Especially, signal interference between macro- and femtocells should be analyzed and carefully coordinated, since femtocells are expected to share the same licensed bandwidth with traditional cellular networks for high frequency efficiency.

For research and performance evaluation of cellular networks with femtocells, system-level simulation is one of the most useful methodologies [6]. In this article, we develop a system-level simulator for LTE (-Advanced) networks with femtocell using event-driven programming. The simulator includes resource management functionalities, such as packet scheduler and call admission control (CAC) schemes based on the 3GPP release 10 standard, and models of wireless channel effects, various real- and non-real time traffic patterns, and user mobility. In addition, the simulator is equipped with a graphical user interface (GUI) to facilitate configuration, operations, and observations of the simulations.

We intensively analyze effects of signal interference between macro- and femtocells for downlink communications utilizing the developed simulator. For the purpose, we define four scenarios that should be considered in femtocell deployment and evaluate capacities for both macroand femtocells under the scenarios based on the simulator.

The remainder of the article is organized as follows. Section 'Preliminaries' provides background for wireless resources and femtocell networks in 3GPP LTE
(-Advanced) systems. In Section 'Simulator design and development', we introduce architecture, functionalities, components, and GUI for the proposed simulator. Then, we analyze interference between macro- and femtocells in the LTE system by utilizing the simulator in Section 'Interference analysis'. Finally, Section 'Conclusion' gives a conclusion.

\section{Preliminaries}

In this section, we briefly introduce wireless resources and femtocell networks in 3GPP long term evolution (LTE) system, since we refer 3GPP release 9 (LTE) and 10 (LTEAdvanced) specifications [7-11] for development of the simulator.

\section{General information on 3GPP LTE system}

The LTE system adopts orthogonal frequency division multiple access (OFDMA) and single carrier-frequency division multiple access (SC-FDMA) for downlink and uplink communications, respectively, in order to effectively utilize wireless resources in both time and frequency domains $[9,12]$. In addition, for high scalability, the LTE system can use variable bandwidth, from 1.4 to $20 \mathrm{MHz}$, and support both time division duplexing (TDD) and frequency division duplexing (FDD). Figure 1 shows two types of frame structures for FDD and TDD defined in [8]. In FDD, both uplink and downlink use the type 1 frame structure in Figure 1a through different frequency bandwidths. In contrast, in TDD, sub-frames in the type 2 frame structure are alternatively used for uplink and downlink transmissions. For transition between uplink and downlink, sub-frames 1 and 6 contain downlink and

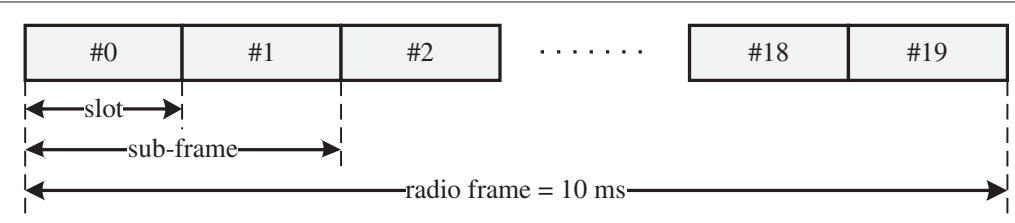

(a) Type 1 for FDD

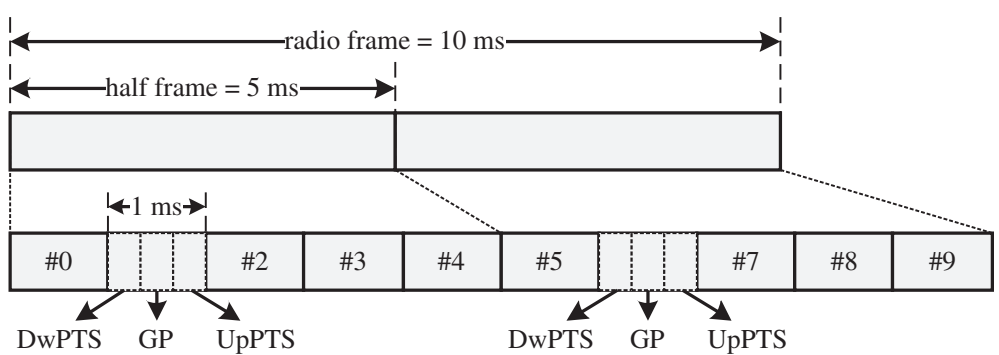

(b) Type 2 for TDD

Figure 1 Frame structures in LTE system. 
uplink pilot time slots (DwPTS and UpPTS) and guard period (GP), as shown in Figure $1 \mathrm{~b}$.

For efficient resource management and scheduling, the LTE system defines physical resource block (PRB), which consists of 12 sub-carriers in the frequency domain and one slot in the time domain as shown in Figure 2. In the case of using normal cyclic prefix (CP) and extended $\mathrm{CP}$ in the LTE physical layer, since a slot consists of seven and six OFDMA symbols, there are $84(=12 \times 7)$ and 72 $(=12 \times 6)$, respectively, resource elements in a PRB. Four resource elements in each PRB are used for downlink reference symbols. By interpolating and averaging reference symbols over multiple reference symbols, the user equipment (UE) can estimate the channel condition between the UE and the base station, so called evolved Node B (eNB). For individual UE, appropriate modulation and coding rates are applied to allocate data bits in PRBs in order to guarantee maximum allowable bit error rate (BER) based on the channel condition. This technique is termed adaptive modulation and coding (AMC). Because of AMC, functionalities for wireless resource management in LTE system, such as packet scheduler and call admission controller, should consider not only conventional metrics but also varying capacities of wireless links for UEs.

\section{Femtocell networks and interference cases}

Home evolved Node B (HeNB), which is a femtocell base station, and expanded evolved UMTS terrestrial radio access network (E-UTRAN) architecture are defined to support femtocells in the LTE-Advanced system [8]. HeNB includes most functionalities of eNB and is connected to cellular core networks through the existing Internet access and HeNB gateway.

In LTE system, wireless resources in both time and frequency domains can be commonly or separately allocated to macro- and femtocells. If macro- and femtocells are allocated to different wireless resources, there exists no signal interference between the macro- and femtocells. However, in this case, utilization of the wireless resources may low, since the wireless resources should be segmented into small pieces. Therefore, many researchers consider reuse of wireless resources in macro- and femtocells in order to improve utilization of the expensive wireless resources [13].

When wireless resources are commonly utilized by macro- and femtocells, signal interference can occur between macro- and femtocells and between different femtocells. Figure 3 shows possible cases of cross-tier interference between macro- and femtocells. In downlink communications, HeNB interferes with signals transmitted from eNB to macrocell UEs (MUEs) ((1) in Figure 3a) while eNB interferes with those from HeNBs to femtocell UEs (FUEs) ((2) in Figure 3a). Conversely, in uplink communications, MUEs and FUEs, respectively, interfere with signals transmitted from FUEs to HeNBs and from MUEs to eNB as (3) and (4) in Figure 3a. Figure 3b shows possible cases of co-tier interference between different femtocells. In downlink communications, HeNBs give interference to signals transmitted from HeNBs to FUEs in other femtocells as (5) and (5') in Figure 3b. On the other hand, in uplink communications, FUEs interfere with signals transmitted from FUEs to HeNBs in different femtocells as (6) and (6') in Figure 3b.

\section{Simulator design and development}

In the developed system-level LTE simulator, we try our best to emulate various functions in the 3GPP LTE (-Advanced) standard. For this purpose, we include packet scheduling and call admission control (CAC) functionalities based on $[7,8]$. In addition, we model wireless channels with multi-path and shadowing effects, four kinds of real time and non-real time IP traffics, and mobility of UEs, in order to evaluate performance of femtocells under realistic environments. Moreover, the simulator adopts a GUI that helps operators of the simulator to set and update simulation parameters easily, to apply arbitrary scenarios using a mouse or trackball, and to observe simulation status and results in real time.

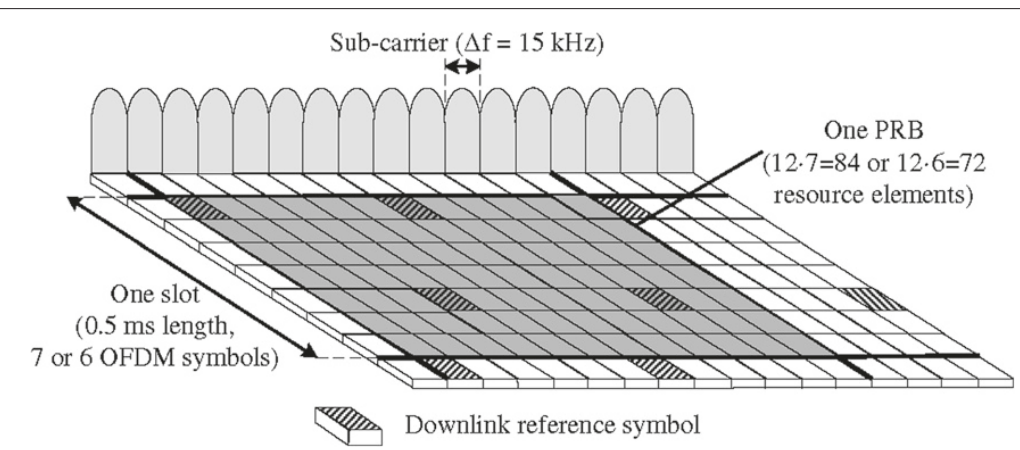

Figure 2 Physical resource block in LTE system. 


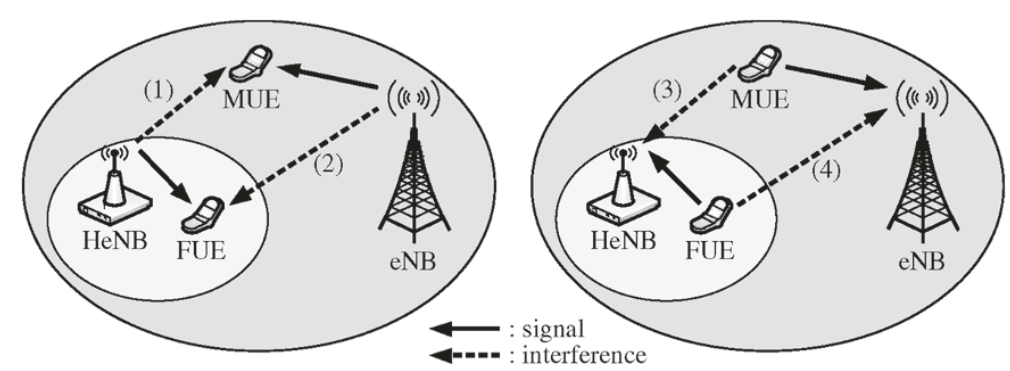

(a) Cross-tier interference
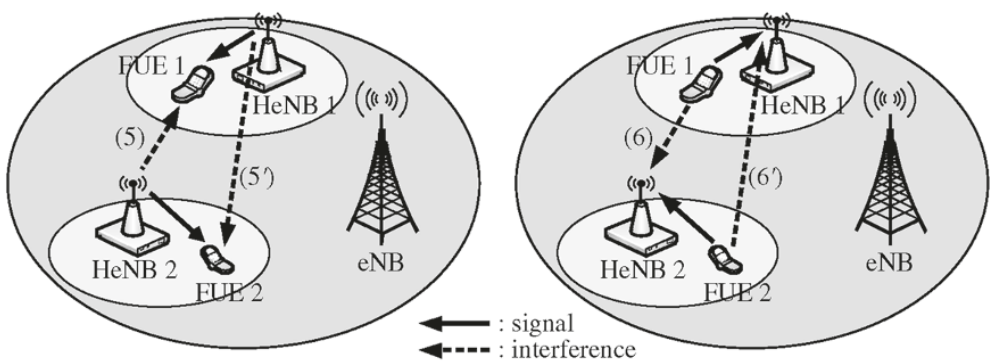

(b) Co-teer interference

Figure 3 Possible cases of signal interference in LTE-femtocell networks.

The simulator is composed of operational and functional parts as shown in Figure 4. The operational part initializes, manages, and finalizes simulations by utilizing initialization, event processing, data management, and finalization modules. The functional part provides various functions and models that are used to emulate LTE network environments, including femtocells and UEs. For the purpose, the functional part consists of five modules that perform packet scheduling and call admission control functionalities and model various wireless channel effects, real and non-real time traffics, and user mobility. Functional modules are tightly integrated with the event process module in the operational part, and they are managed and used in an event-driven manner.

\section{Functional part}

Modules in the functional part can be classified into resource management modules and modeling modules. Resource management modules are used to emulate functionalities related to wireless resources in the LTE system. These resource management modules enable the simulator to evaluate capacity in macro- and femtocell networks. Conversely, modeling modules help the simulator to consider realistic network environments by modeling wireless channel, traffic patterns, and user mobility.

\section{Packet scheduler module}

Packets in the transmit packet buffers in a base station, e.g., eNB and HeNB, are scheduled into available PRBs for wireless transmissions by using the packet scheduler module, as shown in Figure 5. Well-known packet scheduler algorithms, i.e., round robin (RR), proportional fair (PF) [14], and maximum carrier-to-interference ratio (MAX C/I) [15], are included in the simulator to evaluate performance under different types of packet schedulers. We use PF scheduler for packet scheduling in both eNB and HeNBs in the simulation results in this article.

For downlink packet transmissions, PF scheduler in a base station allocates PRBs to the associated UEs considering conditions of wireless links from the base station to the UEs. Let $\mathbb{S}_{\mathrm{PRB}}=\left\{k \mid 1 \leq k \leq N_{\mathrm{PRB}}\right\}$ denote a set of PRBs that can be allocated in a scheduling interval for the downlink transmissions, and $\mathbb{S}_{U E}$ denote a set of associated UEs requiring packet scheduling. PF scheduler allocates a PRB $k\left(\in \mathbb{S}_{\mathrm{PRB}}\right)$ to packets for UE $i_{k}^{*}\left(\in \mathbb{S}_{\mathrm{UE}}\right)$ as $i_{k}^{*}=\arg \max _{i \in \mathbb{S}_{\mathrm{UE}}} C_{i, k} / A_{i}$, where $C_{i, k}$ and $A_{i}$ represent currently achievable data rate with PRB $k$ and average data rate, respectively, for UE $i$.

\section{Call admission control module}

Call admission control (CAC), which is one of the radio resource management functions, plays an important role, since it can prevent network congestion and guarantee a specified level of QoS for on-going calls by accepting or rejecting service requests. Both eNB and HeNB can perform the CAC function considering several conditions, such as channel status, QoS requirements for requested 


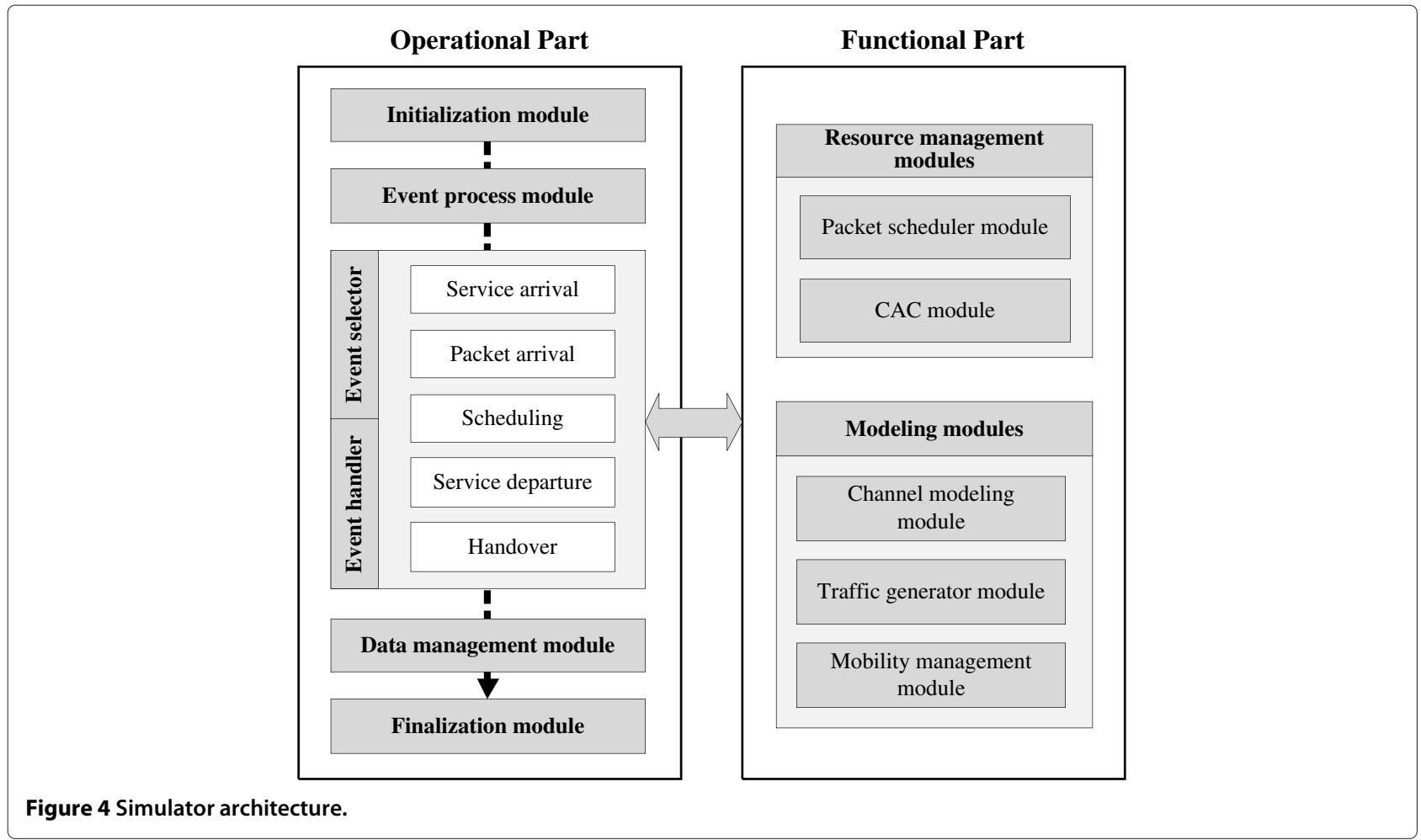

services, buffer state, and so on [7]. In the simulator, we include two CAC algorithms, one accepts a new call based on the estimation of wireless resources [16], and the other decides whether to accept or reject the new call considering the maximum allowed packet delays [17].

\section{Channel modeling module}

This module estimates changes in signal power transmitted through the wireless channel among eNBs, HeNBs, and UEs by utilizing path loss, multi path fading, and shadowing models. We adopt indoor and outdoor path

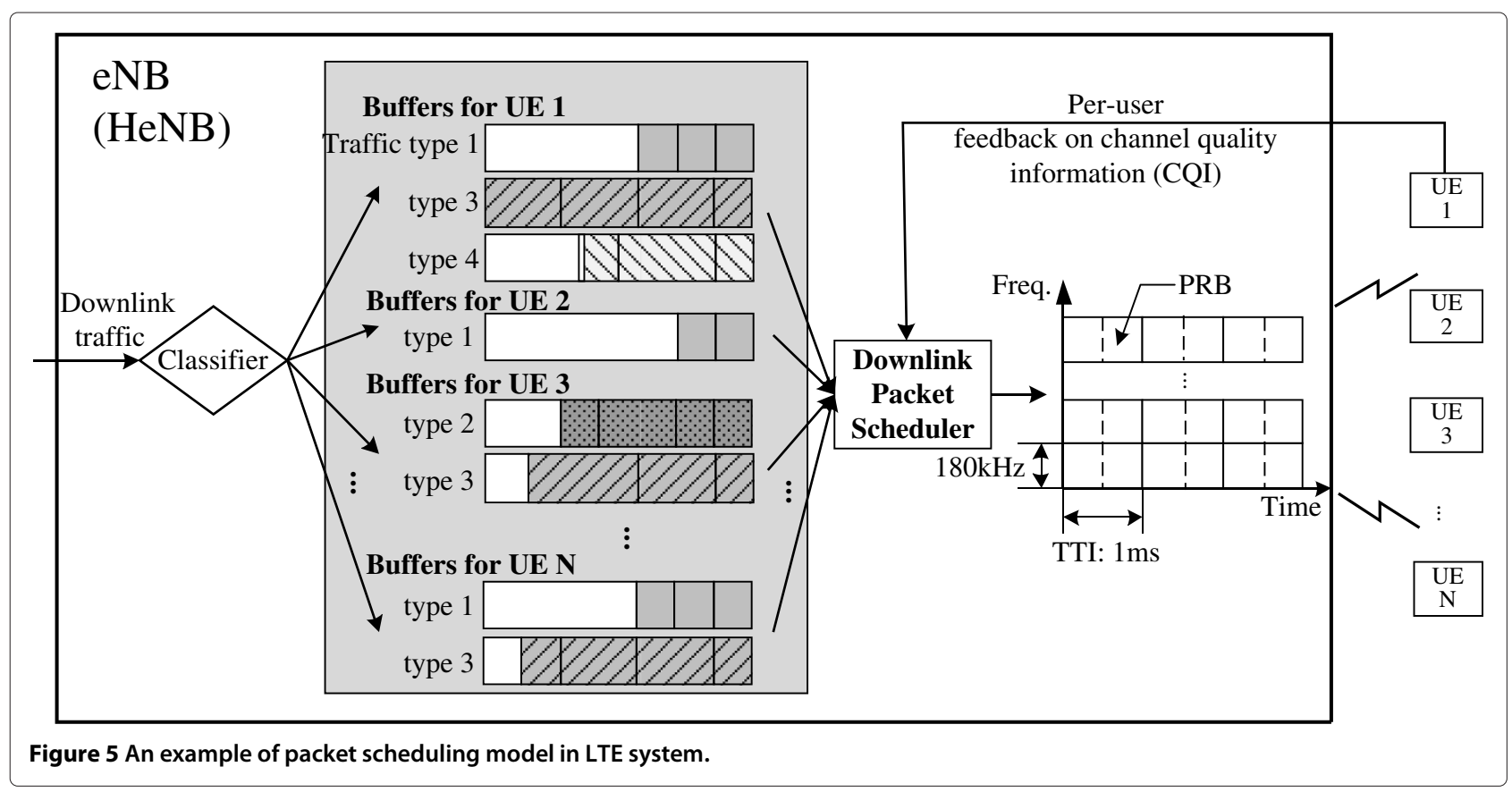


loss models defined in wireless world initiative new radio (WINNER) II project [18] to estimate path loss in macroand femtocell, respectively, as follows:

$$
\mathrm{PL}_{\text {outdoor }}=32.68+35.74 \cdot \log _{10} d \text {, }
$$

$$
\mathrm{PL}_{\text {indoor }}=37.78+36.8 \cdot \log _{10} d \text {, }
$$

where $d$ is distance from the transmitter to the receiver. In addition, the simulator is able to estimate outdoorto-indoor (indoor-to-outdoor) path loss for transmission from eNB to FUEs (from HeNB to MUEs) utilizing Equations (1) and (2). We also consider multi-path fading with an exponential distribution of mean 1 , and shadowing with a normal distribution of zero mean and standard deviation 8 [10].

From the received signal power obtained through the models, the channel modeling module calculates received signal to interference plus noise ratio (SINR) for each wireless link. In order to consider the AMC function in LTE system, the simulator includes discrete spectral efficiency lookup table indexed by SINR [11]. The lookup table defines 35 levels of frequency efficiencies $(\mathrm{bps} / \mathrm{Hz})$ according to the various modulation schemes, i.e., QPSK, 16 QAM, and 64 QAM, and the coding rates under the considerations of 1:2 MIMO, channel prediction, and HARQ. Based on the frequency efficiency determined from the lookup table for each wireless link, the simulator calculates the number of data bits which can be contained in a PRB for the link by multiplying the frequency efficiency with bandwidth $(180 \mathrm{kHz})$ and time duration $(0.5 \mathrm{~ms})$ of the PRB.

\section{Traffic generator module}

In real network environments, mobile users utilize a variety of applications by using their mobile terminals. Therefore, various types of traffic with different characteristics should be transmitted through wireless communication. In the simulator, we build a traffic generator based on stochastic models of four kinds of real- and non-real time traffic types. For real time traffic types, we consider voice over IP (VoIP) for traffic flows of voice conservation and streaming service for those of real time video streaming. In addition, for non-real time traffic types, we consider file transfer protocol (FTP) and web services in order to model traffic flows for burst and intermittent data transmissions, respectively. We refer stochastic models and related parameters in $[19,20]$ to model the traffic types as shown in Table 1.

Upon execution of a service arrival event in Section 'Service arrival event', the traffic generator module

\begin{tabular}{|c|c|c|c|}
\hline $\begin{array}{l}\text { Traffic } \\
\text { type }\end{array}$ & Component & $\begin{array}{l}\text { Stochastic } \\
\text { model }\end{array}$ & Parameters \\
\hline \multirow[t]{3}{*}{ FTP } & File size & Truncated & Mean: 2 MB \\
\hline & & log normal & Std. dev.: $0.722 \mathrm{MB}$ \\
\hline & & distribution & Max: 5 MB \\
\hline Web & Number of & Log normal & Mean: 17 \\
\hline browsing & pages per session & distribution & Std.dev.: 22 \\
\hline \multirow[t]{13}{*}{ (HTTP) } & Main object size & Truncated & Mean: 10710 Bytes \\
\hline & & log normal & Std.dev.: 25032 Bytes \\
\hline & & distribution & Max: 2 MB \\
\hline & & & Min: 100 Bytes \\
\hline & Embedded object & Truncated & Mean: 7758 Bytes \\
\hline & size & log normal & Std.dev. 126168 Bytes \\
\hline & & distribution & Max: $2 \mathrm{MB}$ \\
\hline & & & Min: 50 Bytes \\
\hline & Number of & Truncated & Mean: 5.64 \\
\hline & embedded objects & Pareto & Max: 53 \\
\hline & per pages & distribution & \\
\hline & Reading time & $\begin{array}{l}\text { Exponential } \\
\text { distribution }\end{array}$ & Mean: $30 \mathrm{~s}$ \\
\hline & Parsing time & $\begin{array}{l}\text { Exponential } \\
\text { distribution }\end{array}$ & Mean: $0.13 \mathrm{~s}$ \\
\hline Video & Session & Deterministic & $3600 \mathrm{~s}$ \\
\hline \multirow[t]{13}{*}{ (64kbps) } & duration(movie) & & \\
\hline & Inter-arrival time & Deterministic & $100 \mathrm{~ms}$ \\
\hline & between the & & (based on \\
\hline & beginning of & & 10 frames \\
\hline & each frame & & per second \\
\hline & Number of packets & Deterministic & 8 packets per frame \\
\hline & (slices) in a frame & & \\
\hline & Packet size & Truncated & Mean: 50 Bytes \\
\hline & & Pareto & Max: 250 Bytes \\
\hline & & distribution & \\
\hline & Inter-arrival time & Truncated & Mean: 50 Bytes \\
\hline & between the & Pareto & Max: 12.5 ms \\
\hline & packets in a frame & distribution & \\
\hline \multirow[t]{6}{*}{ VolP } & Average call & Exponential & Mean: $210 \mathrm{~s}$ \\
\hline & holding time & distribution & \\
\hline & Voice CODEC & AMR & $12.2 \mathrm{kbps}$ \\
\hline & Frame length & Deterministic & $20 \mathrm{~ms}$ \\
\hline & Talk spurt length & $\begin{array}{l}\text { Exponential } \\
\text { distribution }\end{array}$ & Mean: 1026 ms \\
\hline & Silence length & $\begin{array}{l}\text { Exponential } \\
\text { distribution }\end{array}$ & Mean: 1171 ms \\
\hline
\end{tabular}

Table 1 Stochastic models for traffic types

generates certain type of traffic as a form of packet arrival event introduced in Section 'Packet arrival event'. Since 
the proposed simulator mainly focuses on the MAC layer functionalities, the higher layers beyond the MAC layer are considered as an abstraction.

\section{Mobility management module}

Consideration of user mobility is very important for realistic simulations, as we have mentioned in Section 'Preliminaries', since movement of UEs yields changes in overall capacity of wireless networks by changing link capacities for UEs. Thus, the simulator includes random walk and random way point mobility models for MUE and FUE, respectively, $[21,22]$. In the random walk model, an UE firstly determines coordinates of the destination based on randomly selected direction, velocity, and flight time, and it starts to move to the destination. This procedure is repeated whenever the UE reaches the destination. In the random way point model, UE is initially located at the random coordinates of the randomly selected room. Then it remains at the location for randomly distributed time durations. After the time duration, it repeats the same procedure.

Mobility management module is also responsible for CQI reports from moving UEs to their corresponding (H)eNBs. 3GPP LTE defines two types of CQI reports according to their reporting interval, i.e., periodic and aperiodic. In addition, according to the type of information contained in the report, it can be classified into wideband and sub-band CQI reports. The wideband CQI report contains a measurement referring to the entire system bandwidth, while the sub-band CQI report includes measurements for various sub-bands. For periodic packet scheduling based on PF scheduler which considers frequency efficiency of each sub-band, the simulator basically adopts the periodic sub-band CQI report.

\section{Event handling}

The simulator was developed based on event-driven programming [23]. Event-driven programming is a program paradigm in which the flow of the program is determined by events. Since event-driven programming can reduce unnecessary loops and operations, it can enhance efficiency and performance of the program and is widely used in well-known network simulators such as OPNET, QualNet, and NS-2 [24-26].

According to event-driven programming, eventprocessing module of our simulator, which is the main loop of the program, is divided into two sections, i.e., event selection and event handling. In addition, event queue based on a linked list data structure is defined to arrange generated events. In event selection, event located at the head of the event queue is loaded and the appropriate event handler is executed based on the type of the event. Consequently, the event handler processes the event using functional modules, global variables, and information contained in the event. The event selection and handling are repeated until the simulation terminates.

Five events are defined in the simulator to manage simulator operations, i.e., service arrival, packet arrival, scheduling, service departure, and handover events.

\section{Service arrival event}

This event indicates arrival of a new service for an UE. Whenever a service arrival event occurs, the event handler executes the CAC module. Then, the CAC module determines whether to accept the service, based on the type of the new service and current system status. If the CAC module decides to accept the service, the simulator successively generates initial and second packet arrival and service departure events for the accepted service, and next service arrival event based on predefined service arrival rate $(\lambda)$, and put those events into the event queue. Conversely, if the CAC module declines the new service, the event handler only generates the next service arrival event and finishes handler operations after putting the event in the event queue.

\section{Packet arrival event}

The packet arrival event means the arrival of a new packet for a specific UE. Since the higher layers beyond MAC layer are considered as an abstraction, the packet arrival is expressed as a form containing attributes of a traffic type, i.e., VoIP, video streaming, FTP, and web service, and of traffic volume in bits. Upon arrival of the event, the event handler commands the traffic generator module to generate an appropriate packet for the service type of the UE. Then, the generated packet is inserted in the UE's transmit packet buffer located at the base station in which the UE is currently connected. After packet generation, the event handler generates a new packet arrival event, based on the next packet arrival time, considering traffic characteristics of the service.

\section{Scheduling event}

The scheduling event indicates execution of the downlink packet scheduler in a specific base station (eNB or HeNB). Currently, uplink packet scheduling is not included in the simulator. The event handler makes the downlink packet scheduler to perform scheduling for packets waiting in the transmit packet buffer, whenever scheduling event occurs. The scheduler first calls the channel module to obtain information on the modulation and coding scheme (MCS) and achievable frequency efficiency (bps/Hz) for each PRB. Based on the information, the scheduler schedules appropriate time and frequency resources (PRBs) for packet transmissions to its associated UEs. The event handler generates a scheduling event for the next scheduling 
time interval and places the event in the event queue after packet scheduling is completed. At this point, by adjusting execution time of the new scheduling event, the simulator can support various scheduling intervals, e.g., multiple of $1 \mathrm{~ms}$.

\section{Service departure event}

The service departure event terminates the service that generated this event. For the purpose, the event handler commands the traffic generator module to initiate variables related to the service. Subsequently, the event handler deletes the packet arrival event for the service to stop packet generation.

\section{Handover event}

The handover event is used to manage mobility of UEs. The event is generated whenever an UE crosses the boundary of the currently located cell. Upon receiving the handover event, the event handler determines if the corresponding UE has ongoing service. If the UE does not receive service, the event handler performs the cell reselection procedure and updates cell ID field in the UE information structure to a new cell ID. On the other hand, if the UE receives a service, the event handler commands the CAC module to determine whether to accept the handover or not. If the new cell cannot accommodate the UE, the service of the UE is terminated, as the same handling procedure for service departure event. However, if the handover of the UE is accepted, as the result of CAC, both cell ID fields in the UE information structure and the next packet arrival event are updated to the new cell ID.

\section{Graphical user interface}

In order to effectively facilitate simulations, the simulator adopts the GUI developed through the Microsoft foundation class (MFC) libraries. By utilizing the GUI, simulation operators can set simulation environments, deploy femtocells, control operation of simulations, and observe simulation results in real time.

The simulator provides two stages for setting of simulation environments, as shown in Figure 6a. In the first stage, operators can set general system parameters, such as simulation duration, carrier frequency, frequency band, and random seed, and parameters related to traffic generation, such as average load and service duration, distribution of service duration, and portion of each service type. Detailed parameters for both macrocell and femtocell base stations and users, including channel effects, number of cells, cell radii, transmission power of eNB and HeNB, and mobility characteristics, can be controlled in the second stage. In addition, type, deployment mode, and transmission power control methods for HeNBs can also be selected in the second stage.
Figure $6 \mathrm{~b}$ shows the main window of the simulator. Network topology and positions of UEs can be observed in real time on the left hand side, while the control of simulation operations and monitoring statistics are enabled using interfaces on the right side of the main window. By clicking the house or building icon in the left hand side of the main window, we can see the indoor environment window for the femtocell as shown in Figure 6c. The floor plan, positions of UEs, and snapshots for SINR and modulation and coding scheme (MCS) levels can be observed in the window.

Brief information about the UE, i.e., ID, velocity, moving direction, and type of ongoing service, can be found by selecting moving UE at both main and indoor environments windows. In addition, by using buttons in the UE information window, received traffic patterns, SINR, and MCS level can be observed via real time graphs. Figures $7 \mathrm{a}$, b show examples of the UE information and the graph on traffic patterns.

\section{Interference analysis}

Performance degradation caused by signal interferences from femtocells is one of the most important issues in femtocell deployments. As we have mentioned in Section 'Preliminaries', femtocells and macrocells may interfere with each other and performance of both networks can be decreased, since many telecommunication companies prefer reusing the frequency bandwidths of existing cellular networks for femtocells due to the frequency efficiency and the cost.

In order to effectively mitigate the performance degradation, both cross-tier interference between macrocells and femtocells and co-tier interference between different femtocells should be analyzed. Thus, we utilize the simulator developed in this article to analyze performance of macro- and femtocell networks. For the analysis, we define four scenarios for different femtocell deployment cases to analyze effects of the distance between femtocell and MUE, the distance between femtocell and macrocell, the number of femtocells, and the distribution of femtocells. Since base stations including eNB and HeNB are major interferers due to their high transmission power, we focus on the downlink performance in the following analysis.

For performance analysis, we assume that macrocell eNB always uses fixed transmission power $(43 \mathrm{dBm})$ for downlink transmissions. Conversely, since transmission powers of femtocell base stations (HeNBs) are closely related to the performance of overall networks [27], we consider three cases for HeNBs' transmission power determination, i.e., fixed transmission power, distance based transmission power determination (D-TPD), and path loss based transmission power determination (P-TPD). In the first case, transmission powers 


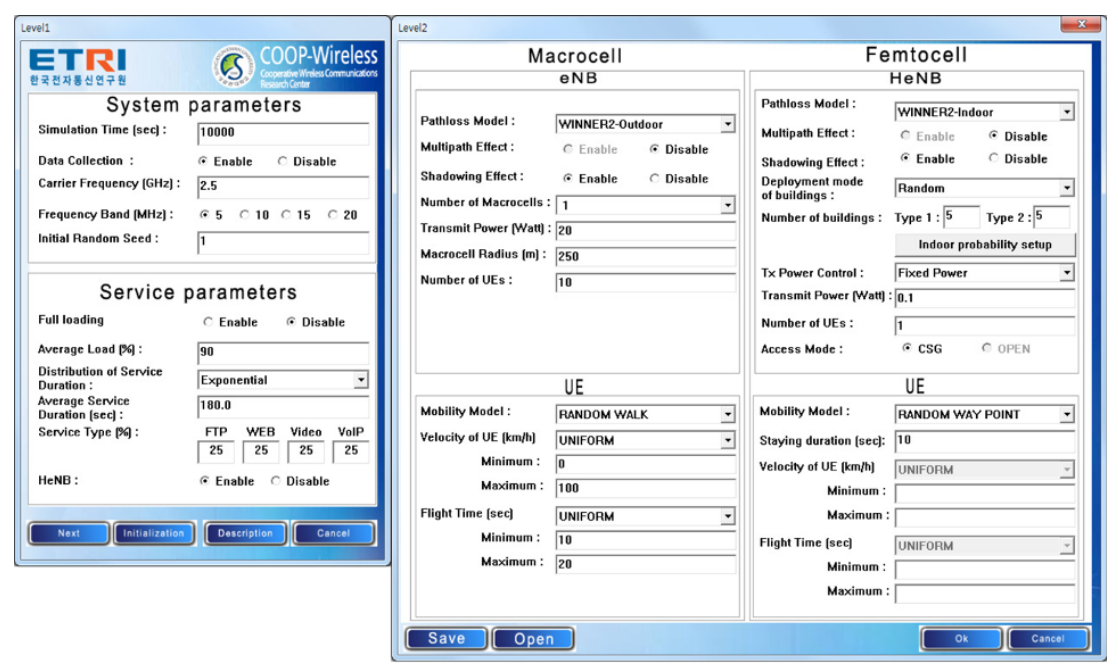

(a) Setting windows for first and second stages

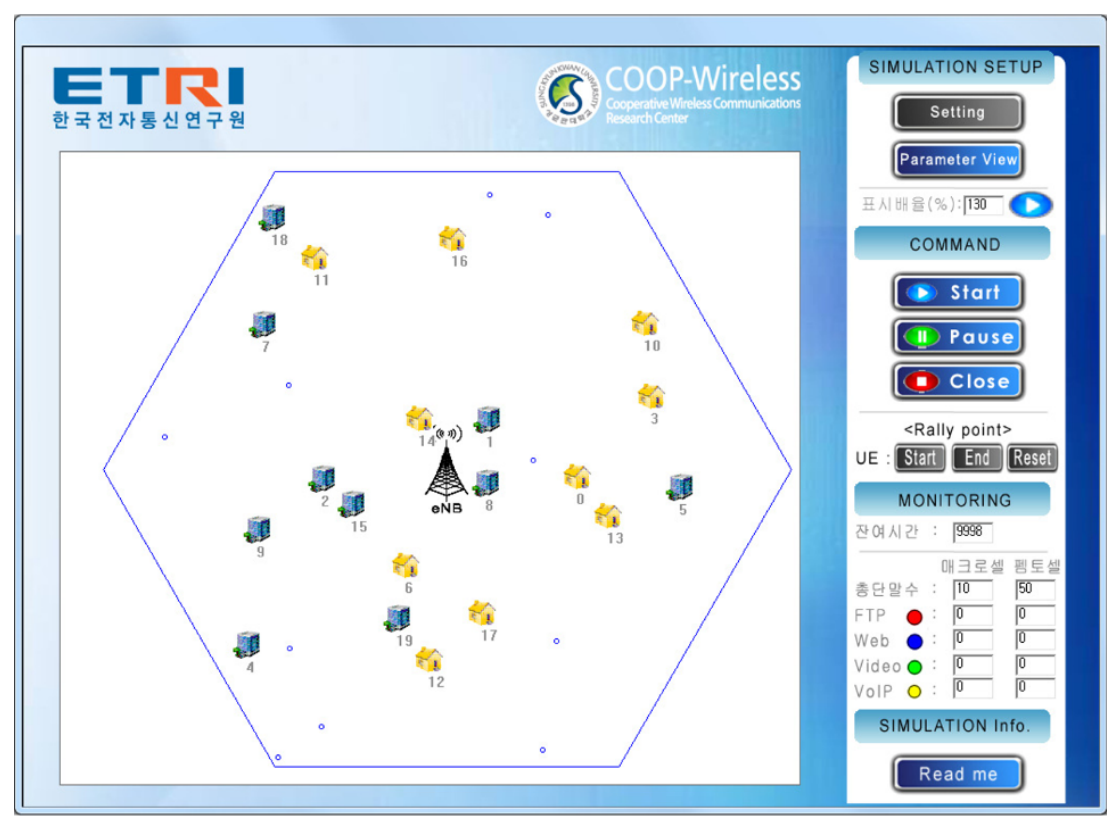

(b) Main window
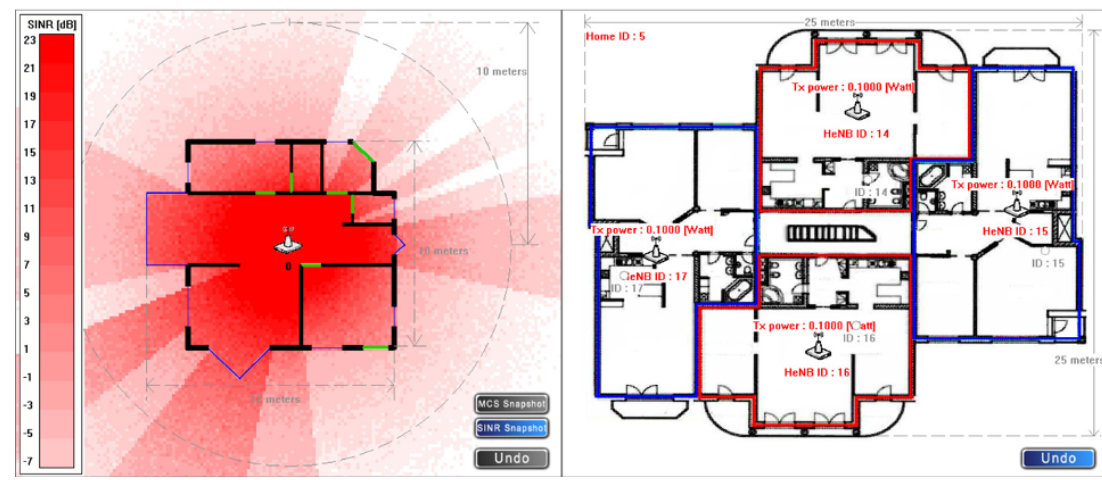

(c) Indoor environments

Figure 6 Graphical user interface of the simulator. 


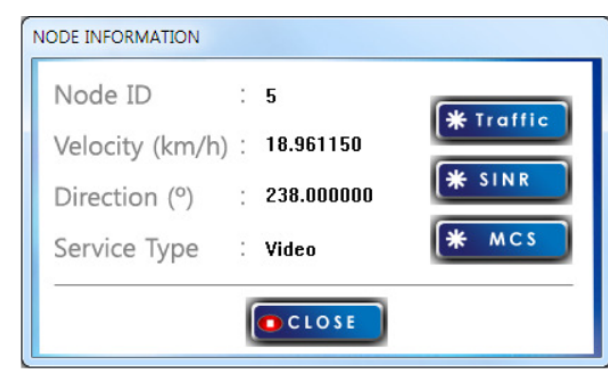

(a) UE information window

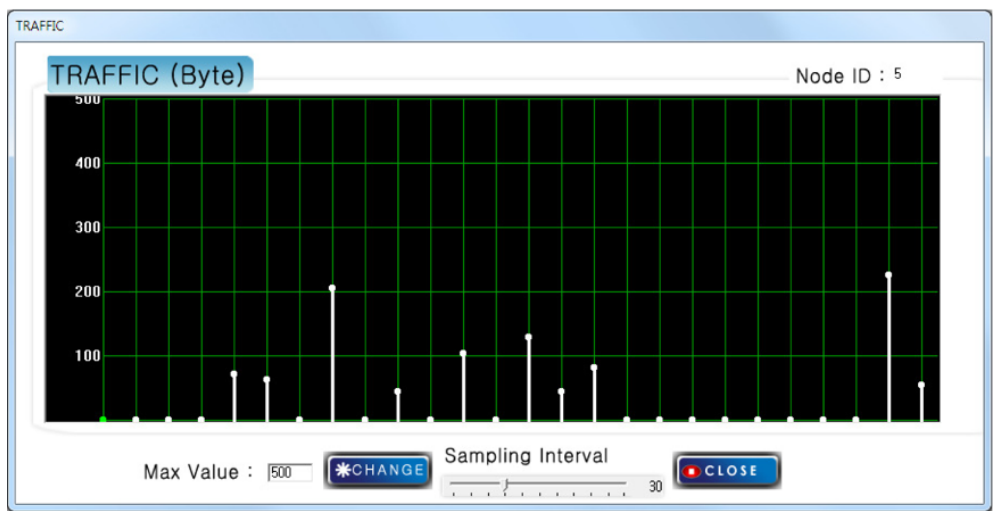

(b) Graph for received traffic patterns

Figure 7 Examples of UE information and graph on traffic patterns.

of HeNBs are fixed to a specific value $(0,10$, and $20 \mathrm{dBm})$, while D-TPD and P-TPD determine transmission powers of HeNBs based on the distances and the path losses among HeNB, MUE, and eNB, respectively, [28].

Assume that a HeNB has recently been installed at an arbitrary location and its power is turned on for the first time. In D-TPD, under the assumption that the HeNB knows distance from the closest eNB, the HeNB decides its transmission power $\left(P^{\mathrm{HeNB}} \mathrm{dBm}\right)$ based on the distance from eNB, $d^{\mathrm{eNB}}$ (meters) [28], as follows:

$$
P^{\mathrm{HeNB}}=\left\{\begin{array}{cl}
20, & 0<d^{\mathrm{eNB}} \leq 85 \\
10, & 85<d^{\mathrm{eNB}} \leq 156 \\
0, & 156<d^{\mathrm{eNB}} \leq 289
\end{array}\right.
$$

On the other hand, in P-TPD, HeNB first discovers the closest macrocell eNB and measures the reference signal received power (RSRP), $R^{\mathrm{eNB}}$, from the eNB to determine its transmission power. If we assume that a MUE near the
HeNB has similar RSRP to the HeNB, the received SINR for the MUE, $S$, can be estimated as

$$
S=R^{\mathrm{eNB}}-\left\{P^{\mathrm{HeNB}}-\mathrm{PL}\left(d^{\mathrm{MUE}}\right)\right\},
$$

where $d^{\mathrm{MUE}}$ (meters) is a distance from the HeNB to the MUE, and $\mathrm{PL}(x)(\mathrm{dB})$ is path loss for the signal interference from the HeNB to the MUE which is $x$ meters apart from the HeNB. In this case, in order to guarantee more than $S_{\text {target }}$ dB SINR for MUEs located farther than $d_{\text {target }}^{\mathrm{MUE}}$ meters, the HeNB determines its transmission power $\left(P_{\text {target }}^{\mathrm{HeNB}}\right)$ from Equation (4) as follows:

$$
P_{\text {target }}^{\mathrm{HeNB}}=\min \left(P_{\max }^{\mathrm{HeNB}}, R^{\mathrm{eNB}}-S_{\text {target }}+\mathrm{PL}\left(d_{\text {target }}^{\mathrm{MUE}}\right)\right),
$$

where $P_{\max }^{\mathrm{HeNB}}$ is maximum transmission power of $\mathrm{HeNB}$. In the simulations, we set $P_{\max }^{\mathrm{HeNB}}, S_{\text {target }}$, and PL $\left(d_{\text {target }}^{\mathrm{MUE}}\right)$ to $20 \mathrm{dBm},-6 \mathrm{~dB}$, and $60 \mathrm{~dB}$, respectively. Table 2 details the parameters used in the simulations.

\section{Scenario 1: effects of the distance between HeNB and MUE} In cellular networks with femtocells, femtocells may reduce capacity of macrocell downlinks by interfering 
Table 2 Simulation parameters

\begin{tabular}{lll}
\hline \multicolumn{2}{c}{ Parameter } & Value \\
\hline \multicolumn{2}{c}{ Carrier frequency } & $2.5 \mathrm{GHz}$ \\
System bandwidth & $10 \mathrm{MHz}$ \\
Scheduling interval & $10 \mathrm{~ms}$ \\
Cell radius & Macrocell & $250 \mathrm{~m}$ \\
& Femtocell & $10 \mathrm{~m}$ \\
Channel models [18] & Outdoor & C1 NLOS \\
& Indoor & A1 NLOS \\
Transmission Power & eNB & $43 \mathrm{dBm}$ \\
& HeNB & $0,10,20 \mathrm{dBm}$ \\
& & for fixed power settings \\
\hline
\end{tabular}

with signals transmitted from eNB to MUE. Moreover, as the MUE gets closer to HeNBs, performance degradation caused by cross-tier interference may increase due to the large RSRP from the HeNBs. In order to analyze effects of the distance between HeNB and MUE, we consider femtocell deployments and the moving path of an MUE as shown in Figure 8. In the scenario, the distance between eNB and MUE is fixed to $100 \mathrm{~m}$, while shortest distance between HeNB and MUE varies from 0 to $20 \mathrm{~m}$ at intervals of $5 \mathrm{~m}$.

Received SINR of MUE according to the movement of MUE in the first scenario is shown in Figure 9a. As shown in the figure, the received SINR increases as the distance between MUE and HeNB increases and as the transmission power of HeNB decreases. When D-TPD is used, since the distances between eNB and HeNBs approximate $100 \mathrm{~m}, 85<d^{\mathrm{eNB}} \leq 156$ and HeNBs set their transmission powers to $10 \mathrm{dBm}$ according to Equation (3). Thus, received SINR for D-TPD is close to that for fixed transmission power with $10 \mathrm{dBm}$. In P-TPD, RSRPs measured at HeNBs for signal from eNB $\left(R^{\mathrm{eNB}}\right)$ are very high, since the HeNBs are closely located with the eNB $(\cong 100 \mathrm{~m})$. Accordingly, HeNBs set their transmission powers to $P_{\max }^{\mathrm{HeNB}}(=20 \mathrm{dBm})$, as Equation (5). Therefore, SINR for P-TPD is similar to that for the case with $20 \mathrm{dBm}$ fixed transmission power.

Figure 9b shows spectral efficiency (bps/Hz) according to the movement of the MUE in Scenario 1. Spectral efficiency is defined as the number of bits that can be transmitted through one Hertz during one second. Trends in Figure $9 \mathrm{~b}$ are similar to those in Figure 9a, since the spectral efficiency is determined from received SINR according to the predefined spectral efficiency lookup table. In addition, we notice that the MUE cannot receive any bits when the MUE is located near HeNB 1, since spectral efficiencies for the MUE become zero due to the strong interference from HeNB 1.

\section{Scenario 2: effects of the distance between eNB and HeNB} Even though distance between MUE and HeNB is fixed, received SINR of an MUE may vary according to the

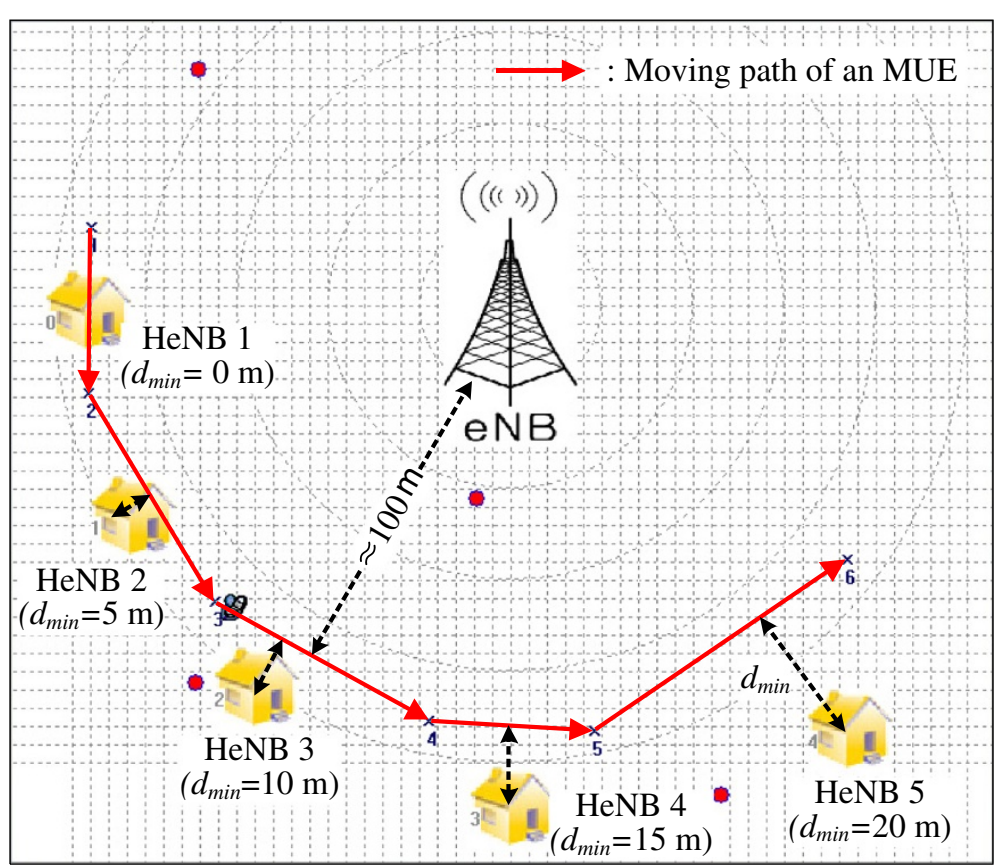

Figure 8 Femtocell deployments and moving path of MUE in Scenario 1. 


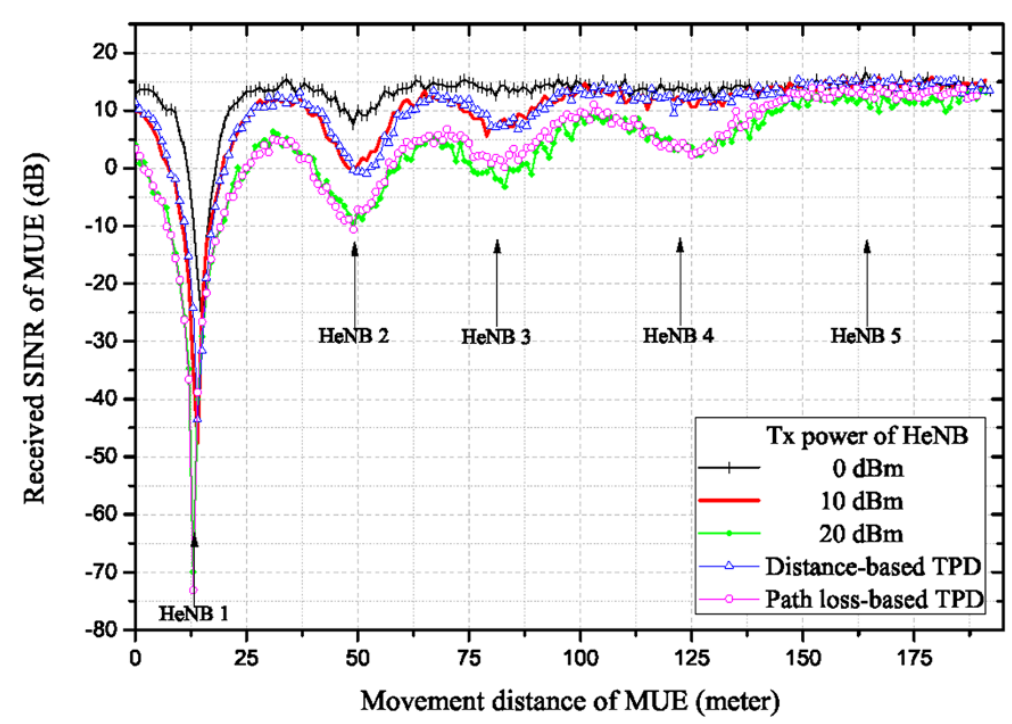

(a) Received SINR

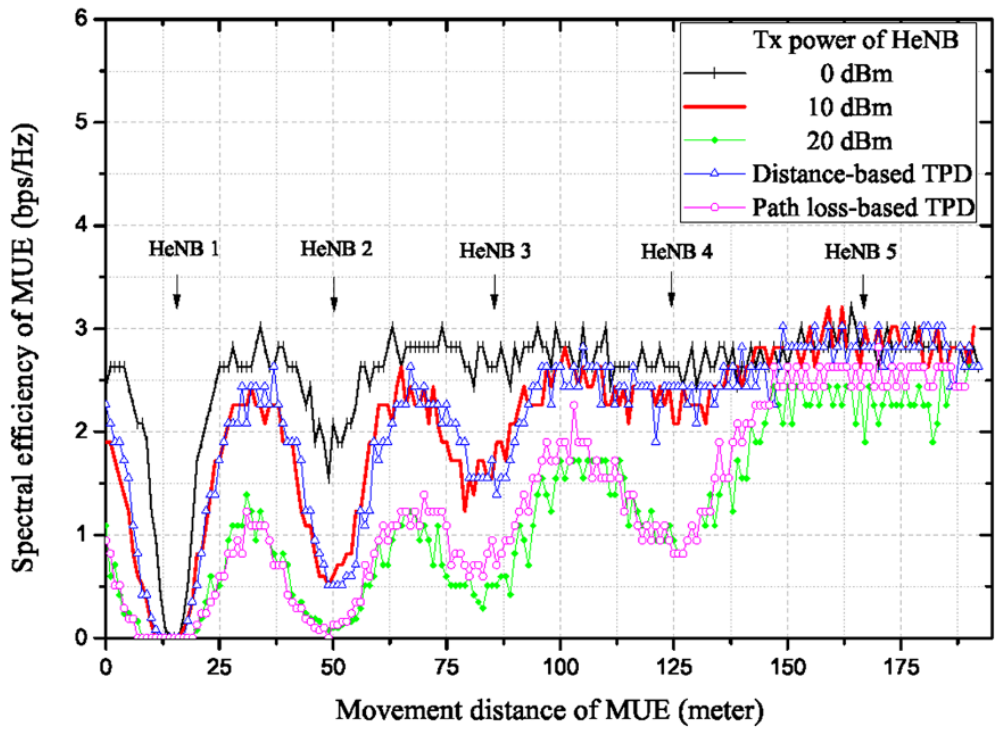

(b) Spectral efficiency

Figure 9 Performance of MUE as varying distance between HeNB and MUE.

distance between the eNB and the HeNB, since received SINR is ratio of received signal to the sum of interferences and noises. In order to analyze the effects of the distance between eNB and HeNB, in the second scenario, we consider femtocell deployments and the moving path of an MUE in Figure 10. In the scenario, distance between eNB and MUE varies from 50 to $250 \mathrm{~m}$ at $50 \mathrm{~m}$ intervals, while the shortest distance from HeNB to MUE is fixed to $10 \mathrm{~m}$.

Figure 11 shows performance of MUE and femtocells based on the location of the MUE. Received SINR and spectral efficiency of the MUE mostly decrease as the distance between eNB and MUE increases. Moreover, whenever the MUE approaches to a HeNB, SINR and spectral efficiency of the MUE further decrease due to the increased signal interference from the HeNB. For D-TPD, HeNB 1 has $20 \mathrm{dBm}$, HeNBs 2 and 3 have $10 \mathrm{dBm}$, and HeNBs 4 and 5 have $0 \mathrm{dBm}$ transmission powers according to Equation (3). Thus, received SINR and spectral efficiency of the MUE in D-TPD are analogous to the results for fixed transmission powers with 20,10 , and $0 \mathrm{dBm}$. Conversely, when transmission powers of HeNBs are determined based on P-TPD as Equation (4), HeNBs 1 and 2 have $20 \mathrm{dBm}$ transmission 


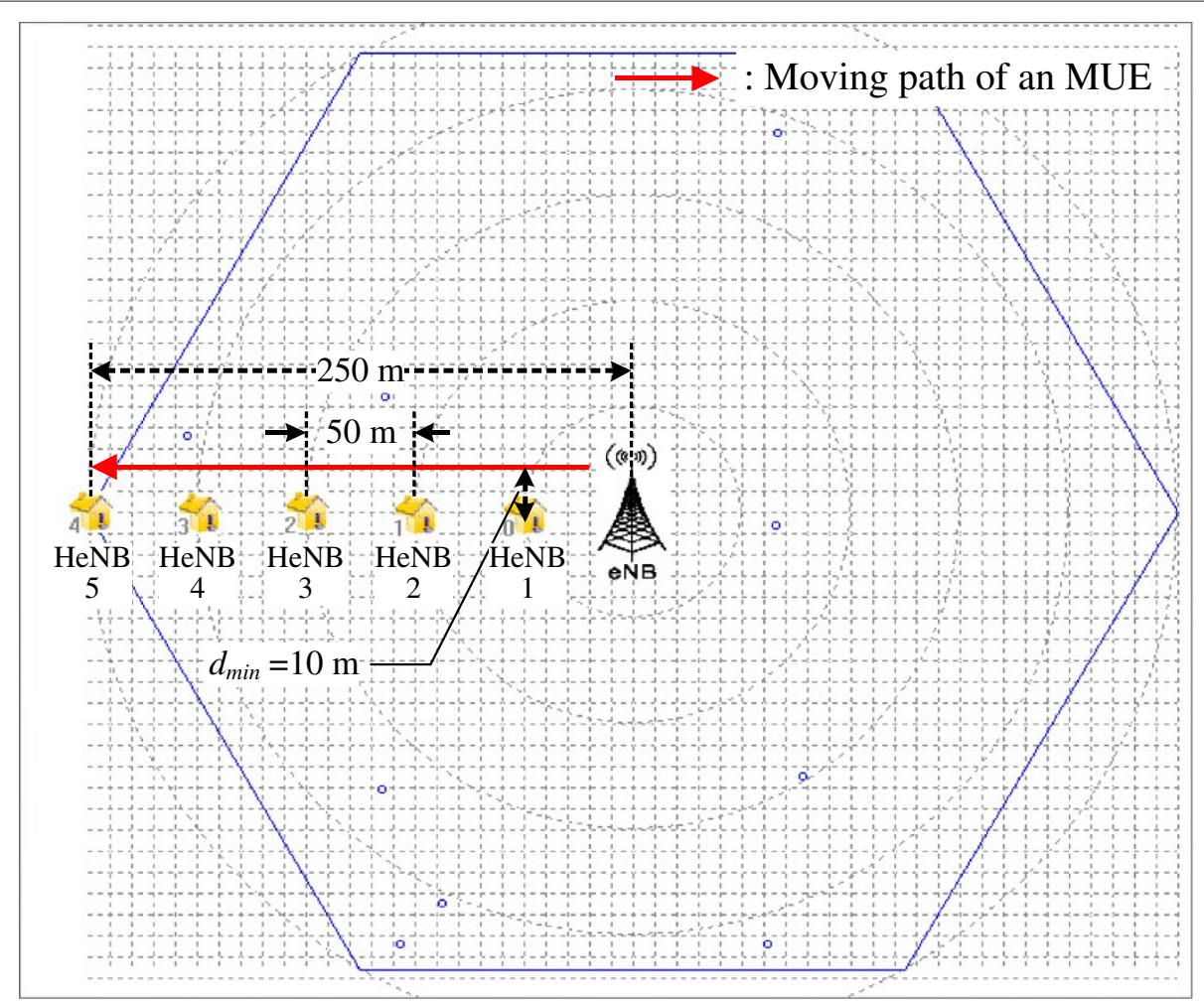

Figure 10 Femtocell deployments and moving path of MUE in Scenario 2.

powers while rest of HeNBs have transmission powers between 10 and $20 \mathrm{dBm}$. Thus, received SINR and spectral efficiency of P-TPD are larger than those for $20 \mathrm{dBm}$ fixed HeNB transmission power, while they are smaller than those for $10 \mathrm{dBm}$ fixed HeNB transmission power.

Table 3 shows average throughput of femtocell as varying the distance from eNB to HeNB. The average throughput of a femtocell is defined as the average of the sums of user throughputs for individual FUEs. When transmission powers of HeNBs are fixed, received interference from eNB to FUE decreases, while the RSRP from HeNB to FUE remains constant as the distance from eNB to FUE increases. Thus, average throughput of femtocells increases as the distances between eNB and HeNBs increase, since the received SINRs of FUEs increase as the eNB recedes from the FUEs. Average throughput of femtocell decreases as the transmission power from $\mathrm{HeNB}$ decreases, for similar reasons.

In both D-TPD and P-TPD, when a HeNB is closely located with the eNB, it uses high transmission power to guarantee certain level of received SINR to its FUEs. On the other hand, if the HeNB is located far from the $\mathrm{eNB}$, it reduces its transmission power to minimize interference to MUEs. From these reasons, average femtocell throughput decreases when distance from the eNB to the
HeNB is larger than 150 and $100 \mathrm{~m}$ for D-TPD and P-TPD, respectively.

\section{Scenario 3: effects of the number of HeNBs}

In Scenario 3, we evaluate performance of an MUE as varying the number of $\mathrm{HeNBs}$ which are located in the vicinity of the MUE. For the purpose, we deploy femtocells and set trajectory of an MUE as shown in Figure 12. The MUE moves on a circle around the eNB in order to maintain constant received power from the eNB. In addition, five groups having different numbers of femtocells (from one to five) are deployed along the path. The minimum distances from each group to MUE and to eNB are set to 20 and $120 \mathrm{~m}$, respectively.

Figure 13 shows received SINR and spectral efficiency according to the MUE's movement. As shown in the figures, both received SINR and spectral efficiency decrease as the number of neighboring femtocells increases. However, we can find that the increase in the number of femtocells affects performance of MUE less than the distance between HeNB and MUE does, compared to the results in Scenario 1. Since the distance between eNB is $120 \mathrm{~m}$, HeNBs set their transmission powers to less than $20 \mathrm{dBm}$ for both D-TPD and P-TPD. Thus, for D-TPD and P-TPD, received SINR and spectral 


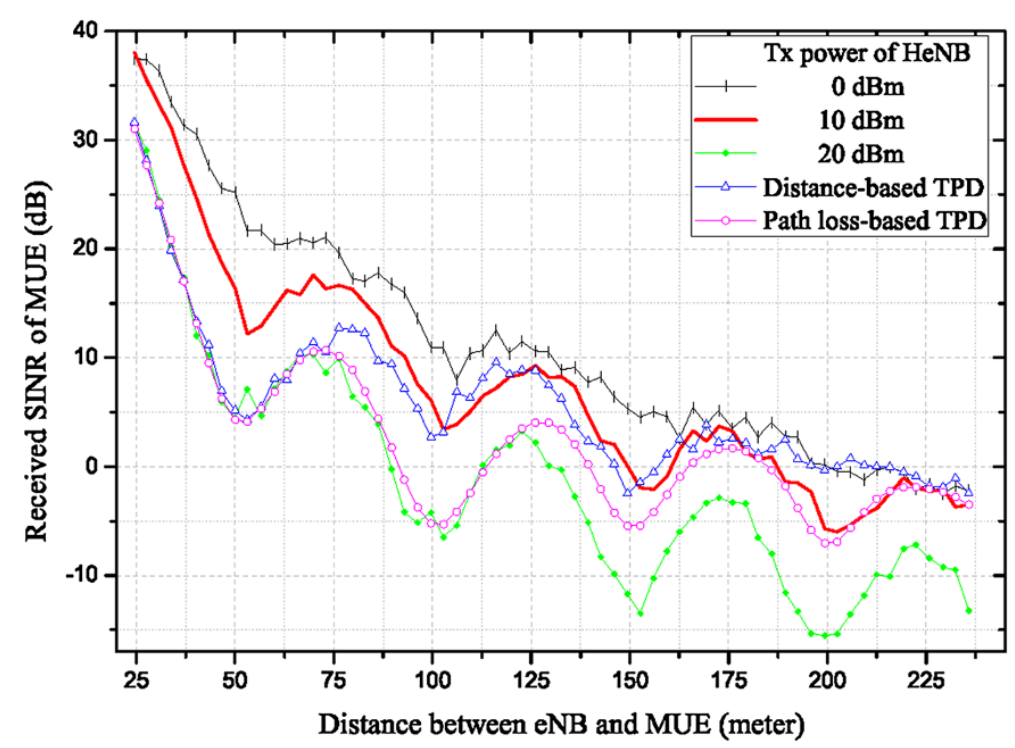

(a) Received SINR of MUE

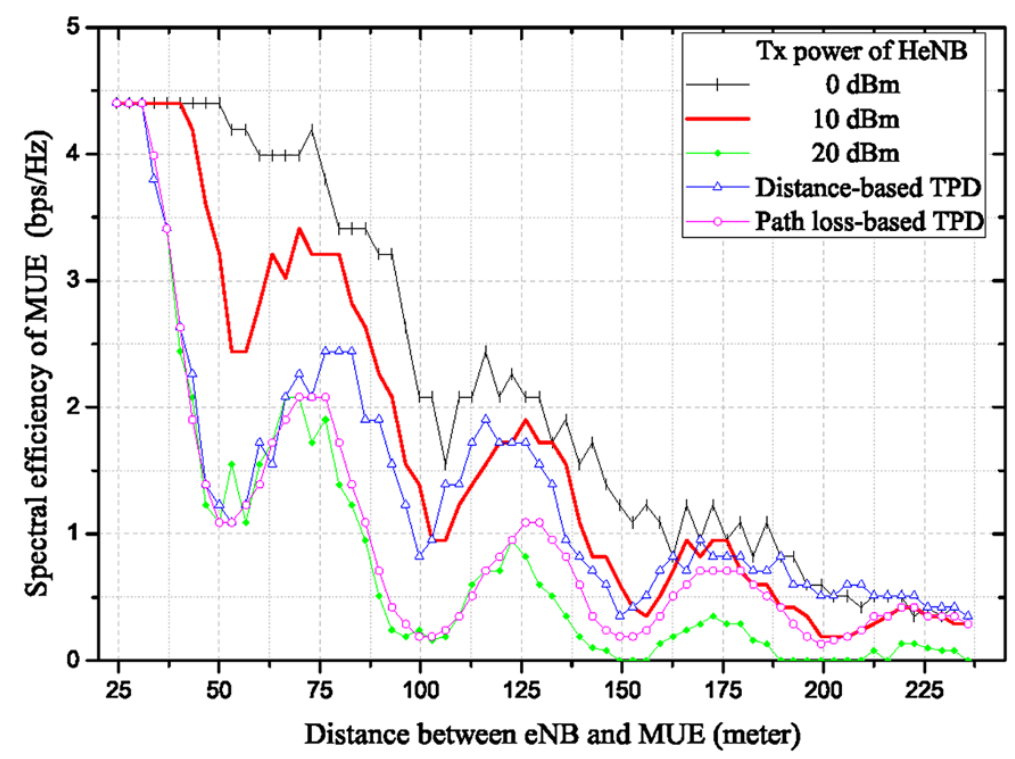

(b) Spectral efficiency of MUE

Figure 11 Performance of MUE as varying distance between eNB and HeNB.

efficiency are better than those for fixed transmission power with $20 \mathrm{dBm}$.

\section{Scenario 4: effects of the distribution of HeNBs}

We evaluate performance of macro- and femtocells under sparse and dense femtocell distributions in Scenario 4. For sparse and dense femtocell distributions, we consider two types of indoor environments which have one and four HeNBs in a building of one story, respectively, as shown in Figure $6 \mathrm{c}$. In addition, four FUEs share wireless resources for communications in each femtocell. In simulations for
Table 3 Average throughput of femtocell (Mbps) according to the distance between eNB and HeNB

\begin{tabular}{ccccccc}
\hline \multicolumn{2}{c}{ Type } & $\mathbf{5 0} \mathbf{m}$ & $\mathbf{1 0 0} \mathbf{m}$ & $\mathbf{1 5 0} \mathbf{~}$ & $\mathbf{2 0 0} \mathbf{~}$ & $\mathbf{2 5 0} \mathbf{~}$ \\
\hline Fixed & $0 \mathrm{dBm}$ & 2.18 & 2.85 & 5.42 & 6.46 & 6.82 \\
& $10 \mathrm{dBm}$ & 3.14 & 8.14 & 11.12 & 12.18 & 12.62 \\
& $20 \mathrm{dBm}$ & 8.09 & 13.53 & 14.73 & 14.6 & 14.7 \\
D-TPD & 7.78 & 8.26 & 11.21 & 6.36 & 7.09 \\
P-TPD & 8.06 & 13.4 & 12.92 & 11.85 & 10.23 \\
\hline
\end{tabular}




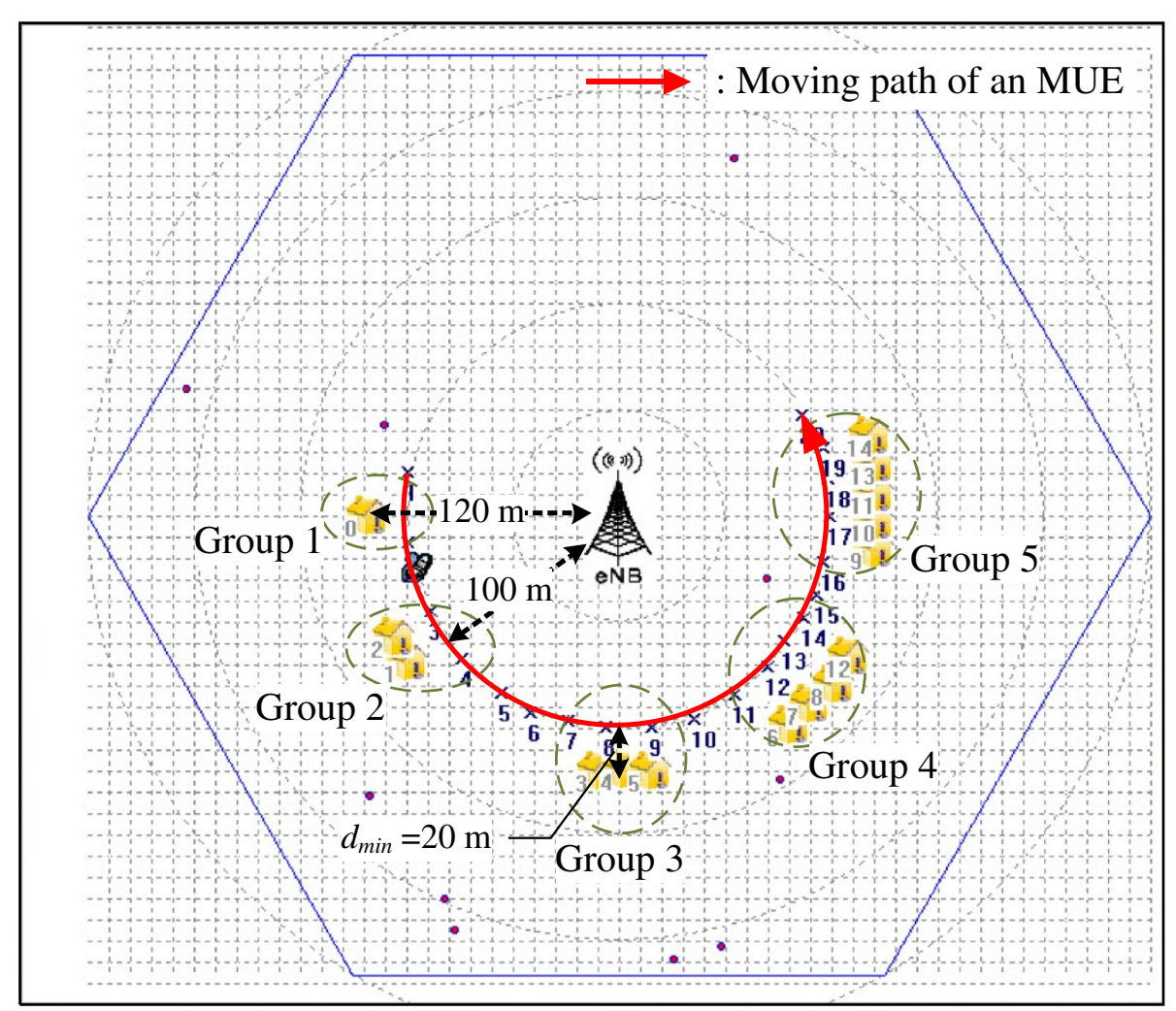

Figure 12 Femtocell deployments and moving path of MUE in Scenario 3.

both sparse and dense femtocell distributions, 50 buildings and 50 MUEs are uniformly deployed in a macrocell. Each building includes one HeNB in sparse femtocell distribution, while it includes four HeNBs in dense femtocell distribution, as shown in Figure 6c.

Table 4 shows average throughputs of macro- and femtocell for sparse and dense femtocell distributions. Throughput of a macrocell is defined as a sum of throughputs of all MUEs in the considered macrocell. If transmission powers of HeNBs are fixed, macrocell throughput decreases as transmission powers of HeNBs increase due to the interference from the HeNBs to the MUEs. In addition, average throughput of a macrocell for dense femtocell distribution is less than that for sparse femtocell distribution, since the total number of femtocells in the dense femtocell distribution is four times larger than that in the sparse femtocell distribution. In comparison with D-TPD and P-TPD, HeNBs in D-TPD generally have lower transmission power than HeNBs in P-TPD, since HeNBs in D-TPD linearly reduces their transmission power, regardless of path loss or channel environments. Therefore, D-TPD has larger average macrocell throughput than P-TPD, because HeNBs with D-TPD less interfere with MUEs.

For HeNBs having fixed transmission powers, femtocell throughput increases as the transmission power increases, since high transmission power improves received SINR and spectral efficiency for FUEs. In dense femtocell distribution, femtocells in a building may interfere with each other, because they share the same frequency bandwidth. Thus, femtocell throughput in the dense femtocell distribution is lower than that in sparse femtocell distribution. In comparison with D-TPD and P-TPD, as we have explained before, HeNBs with D-TPD generally have lower transmission power than those with P-TPD. Thus, P-TPD has larger femtocell throughput than D-TPD as shown in Table 4.

Average throughputs of macro- and femtocells according to the number of femtocells per macrocell are shown in Figures 14a, b, respectively. Since signal interferences from HeNBs are proportional to the number of HeNBs, average throughput of macrocell decreases as the number of HeNBs increases. However, macrocell throughput slowly decreases if HeNBs use low transmission power. Increase in the number of HeNBs also affects the performance of HeNBs, since HeNBs share the same frequency bandwidth and they interfere with one another. However, throughput degradation in a femtocell is much less than that in a macrocell as shown in Figure 14b, because relatively large number of walls may exist between femtocells than between femtocell and macrocell. HeNB with D-TPD reduces its transmission power according to the distance 


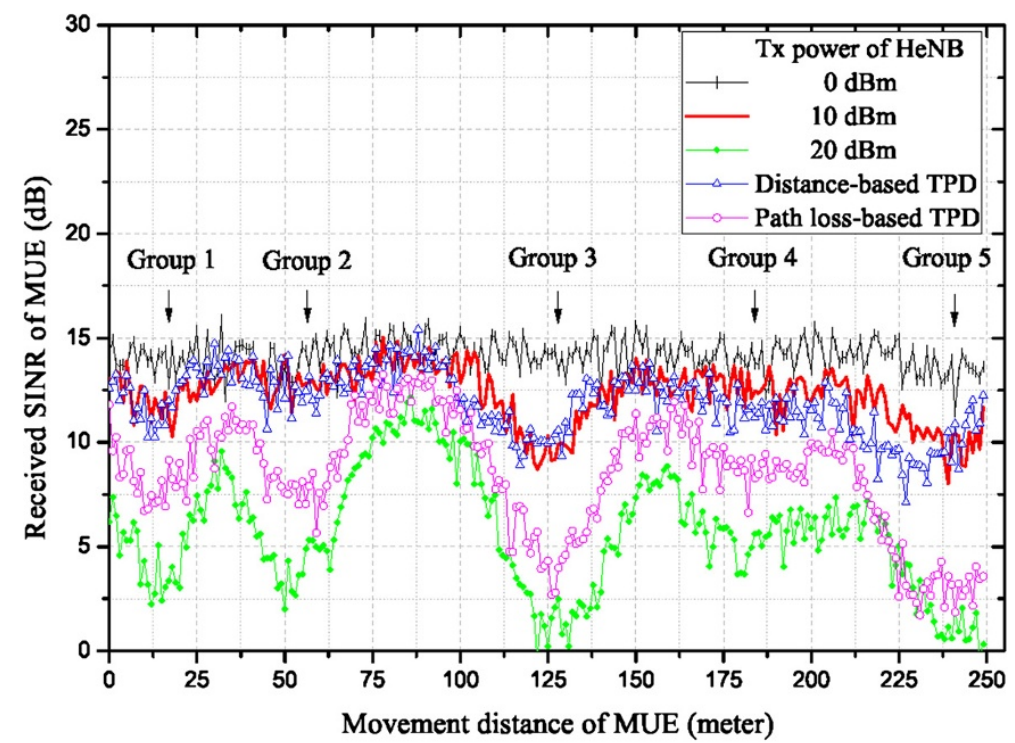

(a) Received SINR

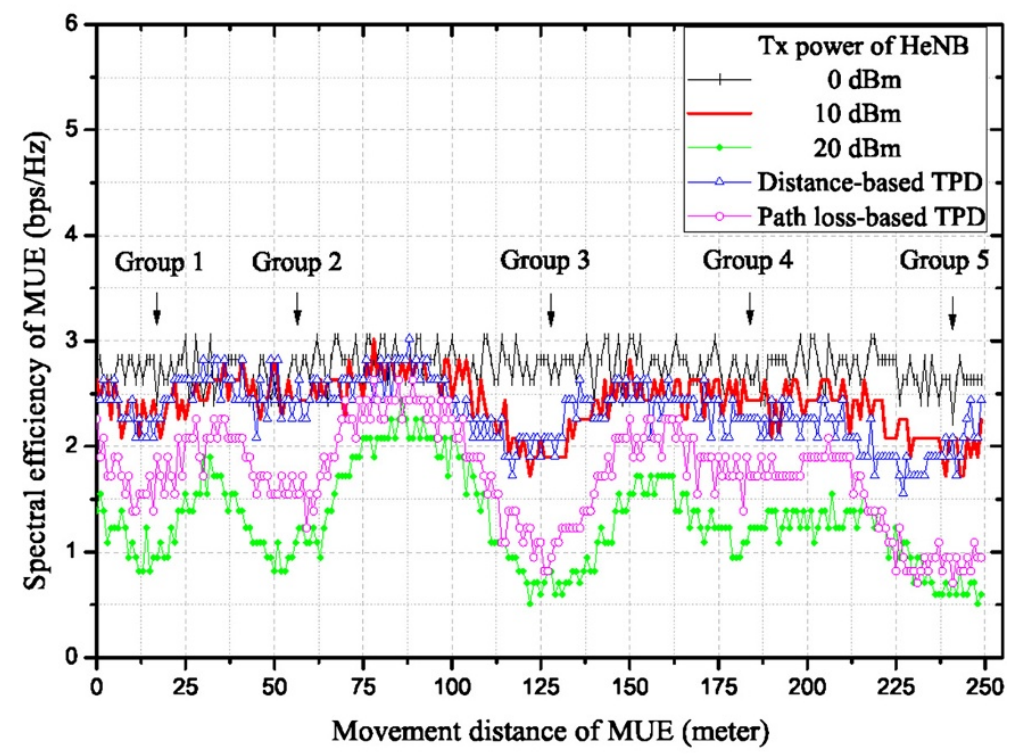

(b) Spectral efficiency

Figure 13 Performance of MUE as varying the number of HeNBs.

from eNB, while HeNB with P-TPD determines its transmission power by considering path loss from the HeNB to an MUE expected to be existed near the HeNB. Due to this characteristic, HeNBs with D-TPD usually have lower transmission power than the HeNBs with P-TPD. Thus, average macrocell throughput for D-TPD is larger than that for P-TPD, and vice versa for the average femtocell throughput.

\section{Conclusion}

Despite the advantages of femtocells, effects of femtocell deployments should be carefully analyzed before their
Table 4 Average throughput of macro- and femtocells (Mbps) under sparse and dense femtocell distributions

\begin{tabular}{rcccccc}
\hline \multicolumn{2}{c}{ Type } & \multicolumn{2}{c}{ Macrocell } & & \multicolumn{2}{c}{ Femtocell } \\
\cline { 2 - 3 } & Sparse & Dense & & Sparse & Dense \\
\hline Fixed & $0 \mathrm{dBm}$ & 6.2 & 5.25 & & 5.52 & 4.31 \\
& $10 \mathrm{dBm}$ & 5.38 & 3.76 & & 9.89 & 6.74 \\
$20 \mathrm{dBm}$ & 3.49 & 1.81 & & 12.69 & 7.87 \\
Distance based & 5.28 & 3.69 & & 8.3 & 6.09 \\
Path loss based & 4.23 & 2.31 & & 10.73 & 7.58 \\
\hline
\end{tabular}




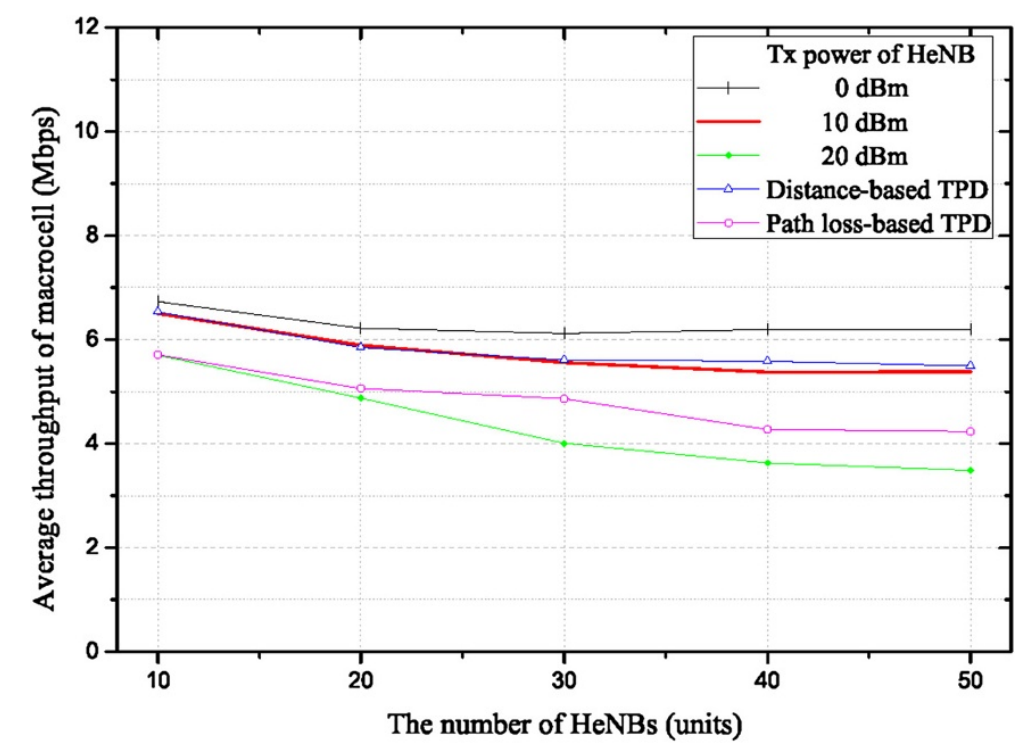

(a) Macrocell

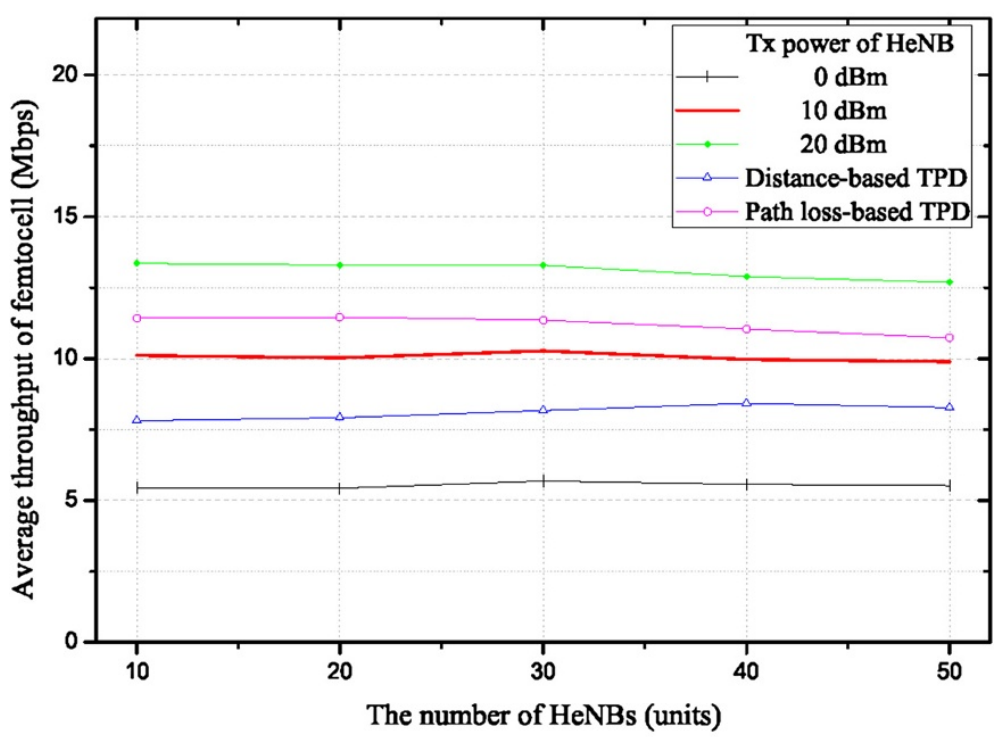

(b) Femtocell

Figure 14 Average throughput of macro- and femtocells as varying the number of femtocells per macrocell.

release, in order to minimize the risks and failures of femtocells in real markets. Thus, we introduced a system-level simulator that can facilitate various simulations for LTE systems with femtocells. For development of the simulator, we described five functional modules, including models of wireless channels, four kinds of IP traffics, and users' mobility, to emulate realistic LTE systems and network environments. In addition, detailed operational events and graphical user interfaces for efficient simulator operations were introduced. We intensively analyzed signal interference between macro- and femtocells under four femtocell deployment scenarios utilizing the developed simulator. From the results, we found that imprudent deployment of femtocells may seriously affect performance of overall networks, and various aspects should be considered in the femtocell deployments. Especially, since femtocells are expected to be arbitrarily deployed by customers, transmission power control of femtocells based on self-optimization manner is important for efficient femtocell deployments. For further studies, by utilizing the developed simulator, we would research on the transmission power control mechanisms which can effectively mitigate cross-tier interference between macroand femtocells by actively adjusting transmission power 
of femtocells based on the close cooperation between eNBs and HeNBs. In addition, various resource partitioning schemes, which statically or dynamically divide radio resources for femto- and macrocells in order to avoid interference and improve overall performance, will be addressed.

\section{Competing interests}

The authors declare that they have no competing interests.

\section{Acknowledgements}

This research was supported by the MKE (The Ministry of Knowledge Economy), Korea, under the ITRC (Information Technology Research Center) support program supervised by the NIPA (National IT Industry Promotion Agency) (NIPA-2012-(H0301-12-1005)) and by the LTE Femtocell research of ETRI (KI002109, 3GPP LTE based All-in-One Femtcell Base Station SoC Platform).

\section{Author details}

${ }^{1}$ College of Information and Communication Engineering, Sungkyunkwan University, Suwon, 440-746, Republic of Korea. ${ }^{2}$ Electronics and Telecommunications Research Institute (ETRI), Daejeon, 305-350, Republic of Korea.

Received: 19 October 2011 Accepted: 24 August 2012

Published: 18 September 2012

\section{References}

1. AT\&T (2010). [http://www.att.com]. Accessed on 15 September (2010)

2. G Mansfield, in Proceedings of the FemtoCells Europe Conference. Femtocells in the US market-business drivers and consumer propositions, (London, UK, p. 2008)

3. V Chandrasekhar, JG Andrews, Femtocell networks: a survey. IEEE Commun. Mag. 46(9), 59-67 (2008)

4. H Claussen, LTW Ho, LG Samuel, An overview of the femtocell concept. Bell Labs Tech J. 13(1), 224-246 (2008)

5. D Calin, H Claussen, H Uzunalioglu, On femto deployment architectures and macrocell offloading benefits in joint macro-femto deployments. IEEE Commun. Mag. 48(1), 26-32 (2010)

6. LChen, W Chen, B Wang, X Zhang, H Chen, D Yang, System-level simulation methodology and platform for mobile cellular systems. IEEE Commun. Mag. 49(7), 148-155 (2011)

7. E-UTRAN overall description state 2 (Release 9). 3GPP TS 36.300, V9.6.0 (2010)

8. E-UTRAN overall description: state 2 (Release 10). 3GPP TS 36.300, V10.2.0 (2010)

9. 3GPP physical channels and modulation (Release 9). 3GPP TS 36.211 V9.1.0 (2010)

10. Physical layer aspect for evolved universal terrestrial radio access UTRA. 3GPP TS 25.814, V7.1.0 (2006)

11. Radio frequency (RF) system scenarios. 3GPP TR 36.942, V9.2.0 (2009)

12. A Ghosh, R Ratasuk, B Mondal, N Mangavedhe, T Thomas, LTE-Advanced: next generation wireless broadband technology. IEEE Wirel. Commun. Mag. 17(3), 10-22 (2010)

13. D Lopez-Perez, A Valcarce, G Roche, Z Jie, OFDMA femtocells: a roadmap on interference avoidance. IEEE Commun. Mag. 47(9), 41-48 (2009)

14. B Al-Manthari, H Hassanein, N Nasser, Packet scheduling in $3.5 \mathrm{G}$ high-speed downlink packet access networks: breath and depth. IEEE Netw. 21(1), 41-46 (2007)

15. HJ Zhu, RHM Hafez, Scheduling schemes for multimedia service in wireless OFDM systems. IEEE Wirel. Commun. 14(5), 99-105 (2007)

16. SJ Bae, B Choi, JJ Lee, S Kwon, MY Chung, in Proceedings of International Conference on Computational Science and Applications (ICCSA 2009) A resource-estimated call admission control algorithm in 3GPP LTE system (Suwon, Korea, 2009)) pp. 250-260

17. SJ Bae, B Choi, JJ Lee, S Kwon, MY Chung, in Proceedings of International Technical Conference of IEEE Region 10 (TENCON 2009). Delay-aware call admission control algorithm in 3GPP LTE system, (Singapore, 2009)) pp. $1-6$

18. P Kyosti, et al., WINNER II channel models. IST-WINNER D1.1.2: V1.1 (2007)
19. WiMAX system evaluation methodology. WiMAX Forum: V2.01 (2007)

20. Next generation mobile networks radio access performance evaluation methodology: V1.2 Next Generation Mobile Networks (NGMN) (2008)

21. T Camp, J Boleng, V Daview, A survey of mobility models for ad hoc network research. Wirel. Commun. Mob. Comput. 2(5), 483-502 (2002)

22. J Broch, DA Maltz, DB Johnson, YC Hu, J Jetcheva, in Proceedings of ACM Mobicom '98. A performance comparison of multi-hop wireless ad hoc network routing protocols, (Dallas, Texas, USA, 1998)) pp. 90-100

23. S Ferg, Event-driven programming: introduction, tutorial, history. [http:// eventdrivenpgm.sourceforge.net]. Accessed on 29 January (2013)

24. Network Performance Management Solutions. [http://www.opnet.com]. Accessed on 19 September (2010)

25. Scalable Network Technologies:QualNet Network Evaluation Software. [http://www.scalable-networks.com]. Accessed on 29 January (2013)

26. The Network Simulator, NS-2. [http://www.isi.edu/nsnam/ns]. Accessed on 22 September (2010)

27. C Chandrasekhar, M Kountouris, JG Andrews, Coverage in multi-antenna two-tier networks. IEEE Trans. Wirel. Commun. 8(10), 5314-5327 (2009)

28. White paper: interference management in OFDMA femtocells. Femto Forum (2010)

doi:10.1186/1687-1499-2012-287

Cite this article as: Bae et al:: Femtocell interference analysis based on the development of system-level LTE simulator. EURASIP Journal on Wireless Communications and Networking 2012 2012:287.

\section{Submit your manuscript to a SpringerOpen ${ }^{\mathcal{O}}$ journal and benefit from:}

- Convenient online submission

- Rigorous peer review

- Immediate publication on acceptance

- Open access: articles freely available online

- High visibility within the field

- Retaining the copyright to your article

Submit your next manuscript at $\gg$ springeropen.com 